\title{
EL USO DEL CINE EN EL CONOCIMIENTO DE LA DISCAPACIDAD: PROPUESTA DIDÁCTICA EN EL GRADO DE MAESTRO
}

\author{
Asunción Lledó Carreres \\ Universidad de Alicante \\ asunción.lledo@ua.es \\ Alejandro Lorenzo Lledó \\ Universidad de Alicante \\ alejandro.Iorenzo@ua.es \\ Elena Pérez Vázquez \\ Universidad de Alicante \\ elena.pv@ua.es \\ Alba Gilabert Cerdá \\ Universidad de Alicante \\ agc136@alu.ua.es \\ Gonzalo Lorenzo Lledó \\ Universidad de Alicante \\ glledo@ua.es
}

Fecha de Recepción: 1 Mayo 2019

Fecha de Admisión: 25 Septiembre 2019

\section{RESUMEN}

En este nuevo marco curricular el profesorado universitario asume nuevos retos metodológicos que nos hagan reflexionar sobre como formar personas competentes y críticas con actitudes de respeto, solidaridad y con puntos de mira abiertos y flexibles. Con la irrupción de las tecnologías de la información y de la comunicación se ha producido cambios importantes en el ámbito metodológico que han afectado a la forma de enseñar con los medios, uno de estos medios es el cine. La inclusión del cine en las metodologías docentes universitarias puede significar una innovación pedagógica que rompa con el tradicional modelo pasivo y de transmisión y dé protagonismo al alumnado para que cree su propio conocimiento. Desde esta perspectiva, el objetivo de este estudio ha sido utilizar el cine como recursos metodológicos para trabajar en asignaturas del Grado de Maestro de Educación Infantil y Primaria contenidos sobre discapacidad. En la acción formativa realizada han participado 156 estudiantes de segundo curso (61 del Grado de Infantil y 95 del Grado de Primaria). Se ha implementado una propuesta didáctica mediante una selección de películas en función de cada discapacidad y una guía de trabajo previa al visionado de la película y posterior de la misma. Se diseñó una rúbrica de autoevaluación de la actividad realizada. La propuesta didáctica planifica- 


\section{EL USO DEL CINE EN EL CONOCIMIENTO DE LA DISCAPACIDAD: PROPUESTA DIDÁCTICA EN EL GRADO DE MAESTRO"}

da ha puesto de manifiesto no solo la adquisición de contenidos referidos a las discapacidades trabajadas sin también creencias favorecedoras hacia la misma, así como también niveles de satisfacción muy positivos con la metodología utilizada con la inclusión del cine en el proceso de enseñanza y aprendizaje.

Palabras clave: discapacidad, cine, recurso, innovación pedagógica.

\section{ABSTRACT}

"The use of cinema in the knowledge of disability: Didactic proposal in the degree of teacher". In this new curricular framework, university teaching staff take on new methodological challenges that make us reflect on how to train competent and critical people with attitudes of respect, solidarity and with open and flexible points of view. With the emergence of information and communication technologies, there have been important changes in the methodological field that have affected the way of teaching with the media, one of these media is cinema. The inclusion of cinema in university teaching methodologies can mean a pedagogical innovation that breaks with the traditional passive and transmission model and gives protagonism to the students so that they can create their own knowledge. From this perspective, the aim of this study has been to use cinema as methodological resources to work on subjects of the Infant and Primary Education Teacher's Degree on disability. 156 second year students (61 from the Infant Grade and 95 from the Primary Grade) took part in the training. A didactic proposal has been implemented through a selection of films according to each disability and a work guide prior to the viewing of the film and after it. A self-evaluation rubric was designed for the activity carried out. The planned didactic proposal has shown not only the acquisition of contents referring to the disabilities worked without also favourable beliefs towards it, as well as very positive levels of satisfaction with the methodology used with the inclusion of cinema in the teaching and learning process.

Keywords: disability, cinema, resources, pedagogical innovation.

\section{INTRODUCCIÓN}

Los nuevos retos metodológicos que se han incorporado en el contexto universitario del siglo XXI, centrados en un aprendizaje más experiencial y significativo que dan un mayor protagonismo al aprendizaje del estudiante, ha supuesto la inclusión de innovaciones metodológicas significativas. A ello, sumamos la influencia de las tecnologías de la información y de la comunicación que han producido cambios importantes en el ámbito metodológico que han afectado a la forma de enseñar con los medios, uno de estos medios es el cine.

La prioridad de esta potencialidad sin dejar de implementar las otras ya indicadas, es por la caracterización de la escuela del siglo XXI como una educación democrática e inclusiva que garantice los principios de igualdad, equidad y justicia social para todos los alumnos (Arnaiz, 2012).

Como consecuencia de asumir dichos retos, los centros escolares tendrán que incardinar dos retos importantes: aprender a convivir y trabajar con la diversidad del alumnado y llevar a cabo una igualdad de oportunidades para todos (Muntaner, Rosello y De la Iglesia, 2016). En base a dicho planteamiento, los futuros docentes deben transformar las aulas en aulas inclusivas con recursos inclusivos que reconozcan a todos y cada uno de su alumnado. Como se indica por la Agenda Educación 2030, "la educación impulsa el desarrollo transformando vidas y para lograrlo, la propia educación debe transformarse: debe estar abierta a todos, ser inclusiva y de buena calidad" (UNESCO, 2017, p.4). Ya que la educación inclusiva representa el camino a seguir para alcanzar sistemas educativos de calidad, justos y equitativos (Arnaiz, De Haro y Maldonado, 2019, p.18). En este escenario que emerge, como plantean Arnaiz, Azorín y García (2015) son dos los ejes que van a pilotar 
la educación contemporánea, la calidad y la equidad, valores que deben considerarse como presupuestos indivisibles y conjugados.

Desde este marco que define la Educación del siglo XIX y junto con la investigación que se presenta y que apuesta por las potencialidades del cine como recurso didáctico que puede conseguir en las aulas un conocimiento mayor de las diferencias individuales, concretamente las referidas a la discapacidad, así como mejorar su inclusión educativa.

A lo largo de la historia del cine se ha podido constatar cómo se ha reflejado la realidad social. Una de esa realidad social también ha estado conformada por la diversidad asociada a discapacidad. Una serie de trabajos (Casado, 2002; Alegre, 2002; 2003; Monjas, Arranz y Rueda, 2005; Merino, 2005; Monjas y Arranz, 2010; Aparicio, 2010; Badía y Sánhez-Guijo, 2010; Herrero y Tovar, 2011; Jiménez, 2014;). El análisis de estos trabajos pioneros en la temática permite identificar películas que han abordado en sus personajes la discapacidad y el tratamiento de la imagen que se percibe en las mismas.

Los resultados de los trabajos de Monjas, Arranz y Rueda (2005) indican que el $64 \%$ de las películas sobre discapacidad se han realizado en el mundo cinematográfico anglosajón y que el 84\% de las películas se ubican en el género drama y que el tipo de discapacidad abordado sería del $34 \%$ sobre la discapacidad intelectual; la discapacidad motora (18\%), las discapacidades sensoriales, tanto visuales (16\%) y auditivas (14\%); mientras que el autismo estaría sobre el $12 \%$. Aunque no se especifican en los resultados una detallada imagen de la discapacidad tratada en las películas sí parece evidente una evolución positiva en los últimos años hacia una visión más positiva de las posibilidades de las personas con discapacidad. Por ello, su tratamiento en el aula puede favorecer un mayor conocimiento y comprensión de la imagen de la discapacidad. Como apuntan Badía y Sánchez-Guijo (2010) los medios de comunicación son de gran influencia de la imagen pública que dan sobre la discapacidad por lo que las producciones cinematográficas reconstruyen en gran medida la percepción que tiene la sociedad de las personas con discapacidad, evolucionando hacia perspectivas más normalizadoras.

Las posibilidades que nos ofrece el cine es favorecer la visibilidad, la normalización y el acercamiento a la realidad y situación de las personas con discapacidad. Es por lo que su principal objetivo ha sido utilizar el cine como recurso metodológico para trabajar en asignaturas del Grado de Maestro de Educación Infantil y Primaria contenidos sobre discapacidad

\section{METODOLOGÍA}

\section{Contexto y participantes}

El contexto en el que se ubica esta investigación es en la formación inicial del título de grado de Maestro en educación Infantil y Primaria. Concretamente en la asignaturas de formación básica de $2^{\circ}$ curso del Grado de Maestro de Educación Infantil "Dificultades de aprendizaje y Trastornos en el desarrollo: respuestas educativas inclusivas" y "Atención a las necesidades Educativas Específicas" de $2^{\circ}$ curso del Grado de Maestro de Educación Primaria. La muestra se ha conformado con 118 estudiantes del Grado de Maestro en Educación Infantil y 112 estudiantes del Grado de Maestro de Educación Primaria, un total de 230 estudiantes de ambos grupos, de los cuales en el Grado de Infantil el 89,25 eran mujeres y el 64,30\% hombres en el Grado de Primaria.

\section{INSTRUMENTO}

Se ha diseñado un guion de análisis de la película para ser utilizado en la actividad mediante el cine y que se presenta en la tabla 1 : 


\section{EL USO DEL CINE EN EL CONOCIMIENTO DE LA DISCAPACIDAD: PROPUESTA DIDÁCTICA EN EL GRADO DE MAESTRO"}

Tabla 1

Guion-análisis de la película

\begin{tabular}{|c|}
\hline Resolvemos a partir del visionado la película \\
\hline -Temática que aborda la película \\
\hline ¿Es apropiada para trabajar los contenidos que aborda? \\
\hline -¿Cómo resolverías las situaciones que plantea la película? \\
\hline -¿Qué películas conoces que aborden dicha temática? \\
\hline -¿Qué emociones ha despertado el visionado de esta película? \\
\hline -¿Qué has aprendido de esta película? \\
\hline -¿Qué te ha gustado más y qué te ha gustado menos? \\
\hline ¿¿Qué opinión os merece la película? \\
\hline -¿Qué se puede aplicar de la película a la realidad actual? \\
\hline
\end{tabular}

Asimismo, se elaboró un cuestionario ad hoc conformado por 11 ítems para conocer el grado de satisfacción con los aprendizajes adquiridos con el uso del cine como recurso de aprendizaje en contenidos sobre discapacidad, con las siguientes categorías de respuesta: $1=$ en total desacuerdo, $2=$ en desacuerdo, $3=$ de acuerdo, y $4=$ totalmente de acuerdo. Se realizó también una pregunta final (ítem 12) en el que se pretendía conocer según los participantes fueran del Grado de Infantil o de Primaria., su predisposición a utilizar cine en las aulas y educar en una herramienta del siglo XIX para educar el siglo XXI (Ambròs y Breu ,2007, p.45)

\section{Procedimiento de la propuesta de intervención}

La propuesta implementada tiene un fundamento normativo contemplado en las s ECI que regulan la verificación de los títulos universitarios oficiales que habilitan para el ejercicio de la profesión de Maestro en Educación Infantil (Orden ECI/3854/2007) y para el Grado de Maestro en Educación Primaria (Orden ECI/38572007). Concretamente la primera reconoce como competencia para el futuro maestro el dominio del uso de diferentes técnicas de expresión, entre las que cabría incluir la audiovisual y conocer las implicaciones educativas de las tecnologías de la información y la comunicación, aunque se cita en particular a la televisión y no el cine. Mientras que en la segunda, se establece como competencia conocer y aplicar en las aulas las tecnologías de la información y de la comunicación y discernir selectivamente la información audiovisual que contribuya a los aprendizajes, a la formación cívica y a la riqueza cultural. Asimismo, en el marco normativo del currículo de la etapa de Educación infantil (Real Decreto 1630/2006, por el que se establecen las enseñanzas mínimas del segundo ciclo de Educación infantil) hace referencia explícita al "acercamiento a producciones audiovisuales como películas, dibujos animados o videojuegos. Mientras que en el currículo de la Educación Primaria (Real Decreto de 2014) en sus diferentes áreas y sus estándares de aprendizaje evaluables, reconoce el cine de animación como un género del cine y menciona el proceso empleado para la creación, montaje y difusión de una película de animación.

Es por ello, que se ha llevado a cabo una propuesta de intervención para desarrollar competencias que permitan desde la formación inicial el uso del cine para ser aplicado en su profesión docente en las aulas de Educación Infantil y Primaria.

Las películas seleccionadas se indican en la tabla 2: 
Tabla 2.

Películas seleccionadas para el uso del cine en el conocimiento de la discapacidad

\begin{tabular}{ll}
\hline \multicolumn{1}{c}{ Película } & \multicolumn{1}{c}{ Temática } \\
\hline $\begin{array}{l}\text { "El pequeño salvaje"(1970) de François } \\
\text { Truffaut. }\end{array}$ & Historia de la discapacidad. \\
"Mi pie izquierdo" (1989) de Jim Sheridan & Discapacidad física \\
"El milagro de Anna Sullivan” (1962) de & Discapacidad auditiva. \\
Arthur Penn, & \\
"Rojo como el cielo" (2007) de Cristiano & Discapacidad visual \\
Bortone & \\
"Adam" (2009) de Max Mayer & $\begin{array}{l}\text { Trastorno del Espectro Autista } \\
\text { (Asperger). }\end{array}$ \\
"León y Olvido" (2004) de Xavier Bermúdez & Síndrome de Down. \\
Me llaman Radio/ Radio (2003) de Mike & Discapacidad intelectual \\
Tollin & \\
"Buscando a Nemo"(2003) de Andrew & Valores diversidad \\
Stanton, Lee Unkrich y Alan Barillaro. & \\
"El pequeño Tate" (1991) de Jodie Foster & Altas capacidades \\
\hline "Vitus" (2006) de Fredi Murer. & Altas capacidades
\end{tabular}

Una vez seleccionadas las películas se realiza el proceso de intervención, en una primera fase la realización a nivel individual con la resolución de cuestiones a partir del visionado de cada película. A continuación y en una segunda fase y siguiendo a Aguilar (1996) se utiliza la técnica del cinefórum se vivencia un proceso de interiorización y de libre expresión a través de la interacción grupal, produciéndose una reflexión crítica que puede generar cambios y nuevas actitudes. Cuestiones que son claves en los contenidos de las materias en las que se ha implementado la propuesta que se presenta.

\section{RESULTADOS}

Se presentan los resultados obtenidos en la muestra de participantes de los dos Grados tanto de Infantil como Primaria (tabla 3): 


\section{EL USO DEL CINE EN EL CONOCIMIENTO DE LA DISCAPACIDAD: PROPUESTA DIDÁCTICA EN EL GRADO DE MAESTRO"}

Tabla 3

Grados de acuerdo con el uso de cine como recurso en contenidos de discapacidad

\begin{tabular}{lccccc}
\hline & \multicolumn{1}{c}{ Media } & \multicolumn{4}{c}{ Categorías de respuesta } \\
Ítems & & 1 & 2 & 3 & 4 \\
\hline $\begin{array}{l}\text { 1. Las películas trabajadas se adecuan a los contenidos } \\
\text { sobre discapacidad de la asignatura. }\end{array}$ & 3.26 & 2.0 & 11.4 & 45.5 & 41.1 \\
$\begin{array}{l}\text { 2. El visionado ha permitido la transmisión de contenidos } \\
\text { y actitudes sobre la discapacidad. }\end{array}$ & 3.52 & .3 & 3.0 & 41.4 & 55.3 \\
$\begin{array}{l}\text { 3. El uso del cine en la asignatura tiene que ser una } \\
\text { actividad complementaria y no básica. }\end{array}$ & 1.48 & 56.5 & 39.5 & 3.3 & 0.7 \\
$\begin{array}{l}\text { 4. Me he sentido motivado con el visionado de películas y } \\
\text { las actividades individuales y el cine fórum. }\end{array}$ & 3.68 & 1.3 & 0.7 & 27.1 & 70.9 \\
$\begin{array}{l}\text { 5. Necesito una formación específica para utilizar el cine } \\
\text { como recurso en las aulas de infantil y primaria. }\end{array}$ & 1.25 & 76.8 & 21.5 & 1.7 & 0.0 \\
$\begin{array}{l}\text { 6. He cambiado bastante mis actitudes ante las personas } \\
\text { con discapacidad después del visionado de la película. }\end{array}$ & 3.63 & 0.3 & 1.7 & 33.1 & 64.9 \\
$\begin{array}{l}\text { 7. El cine fórum ha supuesto una estrategia metodológica } \\
\text { para reflexionar y compartir conocimientos. }\end{array}$ & 3.48 & 0.0 & 3.7 & 44.4 & 51.9 \\
$\begin{array}{l}\text { 8. Tengo conocimiento de la inclusión en el cine en } \\
\text { asignaturas del Grado de Maestro. }\end{array}$ & 2.54 & 13.4 & 37.1 & 31.8 & 17.7 \\
$\begin{array}{l}\text { 9. Tengo conocimiento de la inclusión en el cine en } \\
\text { asignaturas del Grado de Maestro. }\end{array}$ & 1.23 & 80.3 & 17.7 & 1.0 & 1.0 \\
$\begin{array}{l}\text { 10. Las películas visionadas y las actividades realizadas } \\
\text { nos ha generado una mirada más crítica de la realidad. }\end{array}$ & 3.27 & 3.3 & 11.3 & 41.1 & 44.5 \\
$\begin{array}{l}\text { 11. Con el visionado de las películas me han cambiado } \\
\text { los conocimientos que tenía sobre la discapacidad. }\end{array}$ & 3.73 & 0.0 & .7 & 25.4 & 73.9 \\
\hline
\end{tabular}

Tabla 4

Grados de acuerdo con el uso de cine como recurso en las aulas de infantil y primaria

\begin{tabular}{|c|c|c|c|c|c|}
\hline \multirow[b]{2}{*}{ Ítems } & \multirow[t]{2}{*}{ Media } & \multicolumn{4}{|c|}{ Categorías de respuesta } \\
\hline & & 1 & 2 & 3 & 4 \\
\hline \multicolumn{6}{|l|}{ Grado Maestro Educación Infantil } \\
\hline $\begin{array}{l}\text { 12. Estoy predispuesto/predispuesta a utilizar el cine } \\
\text { como recurso didáctico para abordar la inclusión de la } \\
\text { discapacidad en las aulas de infantil cuando sea docente. }\end{array}$ & 3.63 & 0.3 & 1.7 & 33.1 & 64.9 \\
\hline \multicolumn{6}{|l|}{ Grado Maestro educación Primaria } \\
\hline $\begin{array}{l}\text { 12. Estoy predispuesto/predispuesta a utilizar el cine } \\
\text { como recurso didáctico para abordar la inclusión de la } \\
\text { discapacidad en las aulas de infantil cuando sea docente. }\end{array}$ & 3.63 & 2.2 & 2.2 & 10.9 & 84.8 \\
\hline
\end{tabular}

Cabe destacar de los resultados obtenidos altos valores en la categoría de totalmente de acuerdo $(70.9 \%)$ que constata la motivación que ha supuesto para el alumnado participante el uso de este medio audiovisual así como, las potencialidades del cine para determinar cambios en los conocimientos que tenían sobre la discapacidad (73.9\%). De la misma manera aunque en menor grado (64.9\%) su influencia en el cambio de actitudes. En cuanto a la aplicación del cine en su futuro docente, los estudiantes del Grado de Primaria con un 84.8\% muestran una mayor predisposición a la utilización del cine en su futura labor docente. También indicar la no necesidad de formación específica en cine para su utilización $(76.8 \%)$ por lo que facilita su uso. 


\section{CONCLUSIONES}

Los resultados obtenidos en este estudio, muestran grados de acuerdo importantes con las cuestiones planteadas, destacando la motivación y el cambio de conocimientos y actitudes sobre la discapacidad.

Los trabajos de Martínez-Salanova (2002); Alegre (2003); Ambrós y Breu (2007); Breu (2010); Breu y Ambròs, (2011); Martínez-Salanova $(2016,2017)$ ha realizado propuestas didácticas para las aulas de primaria y secundaria en la misma línea del estudio, es decir desde sus potencialidades como recurso para aprender con el cine.

La propuesta didáctica implementada con el uso del cine en los estudiantes del Grado de Maestro de Educación Infantil y Primaria, pionera como manifiestan, ha arrojado resultados que refuerzan las posibilidades educativas de este recurso como trasmisor de contenidos y cambios de actitudes y conocimientos sobre la discapacidad así como la consideración de recurso útil para acercarnos más a la comprensión de la discapacidad. Se concluye por una apuesta sobre las posibilidades que ofrece el cine como recurso para el conocimiento de la discapacidad en la educación en todas sus vertientes y etapas.

\section{REFERENCIAS BIBLIOGRÁFICAS}

Aguilar, P. (1996). Manual del espectador inteligente. Madrid: Editorial Fundamentos

Alegre, 0. (2002). La discapacidad en el cine: propuestas para la acción educativa. Comunicar, 18, 157-161. https://doi.org/10.3916/C18-2002-25

Alegre, 0. (2003). La discapacidad en el cine. Barcelona: Octaedro.

Amar, V. M. (2003). Comprender y disfrutar en el cine. La gran pantalla como recurso didáctico. Huelva: Grupo Comunicar Ediciones.

Ambròs, A., y Breu, R. (2007). Cine y educación: el cine en el aula de primaria y secundaria. Barcelona: Graó.

Aparicio, D., y Gómez-Vela, M. (2010). De criados mudos, jóvenes sordas y otros estereotipos. Las personas con problemas de audición y lenguaje en el cine. Revista de medicina y cine, 6(2), 4752.

Arnaiz, P. (2012). Escuelas eficaces e inclusivas: cómo favorecer su desarrollo. Educatio siglo XXI. Revista de la Facultad de Educación, 30 (1), 25-44.

Arnaiz, P., Azorín, C., y García, M.P. (2015). Evaluación de planes de mejora en centros educativos de orientación inclusiva, Revista de currículum y formación del profesorado, 19 (3).

Arnaiz, P., De Haro, R. (2019). Barreras al aprendizaje y a la participación de futuros profesionales en una escuela inclusiva. NAER, of new approaches in educational research, 8 (1), 19-26. http://DOI:10.7821/naer.2019.1.321.

Badía, M, y Sánchez-Guijo (2010). La representación de las personas con discapacidad visual en el cine. Rev Med Cine, 6(2) 69-77.

Breu, R. (2010). El documental como estrategia educativa. Barcelona: Graó.

Breu, R. y Ambròs, A. (2011). Uso didáctico del documental. Barcelona: Graò.

Casado, R. (2002). La discapacidad en el cine como recurso didáctico-reflexivo para la formación inicial de maestros. Comunicar (18), 163-168.

Jiménez, L.A. (2014). La discapacidad en el cine en 363 películas. Madrid: Fundación ONCE.

Herrero, B. y Tovar, M. (2011). Cine y discapacidad: la construcción de la identidad del otro. Un caso de estudio: la vida secreta de las palabras (ISABEL COIXET, 2005). En I Jornadas de Comunicación y personas con discapacidad, p.33-51. Sevilla: Astigi.

Martínez-Salanova, E. (2002). Aprender con el cine, aprender de película. Una visión didáctica para aprender e investigar con el cine. Huelva: Grupo Comunicar. 
Martínez-Salanova, E. (2016). Cine para educar. Miradas y expectativas didácticas. En E. MartínezSalanova (Eds). Artes y Educación de varios. Sevilla. Universidad Pablo de Olavide.

Martínez-Salanova, E. (2017). Educomunicación. El cine para educar. Los pintores en el cine (I). Aularia: Revista Digital de Comunicación, 6 (2), 1-10.

Merino, M. (2005). La parálisis cerebral en el cine. Rev Med Cine (1), 66-76.

Monjas Ma.., Arranz F. y Rueda E. (2005). Las personas con discapacidad en el cine. Siglo Cero. 36(1), 13-29.

Monjas Ma ${ }^{\mathrm{a}}$., Arranz F. (2010). El cine como recurso para el conocimiento de las personas con discapacidad: Veinticinco películas de la última década. Rev Med Cine, 6(2), 55-68.

Muntaner, J.J., Roselló, R.M. y De la Iglesia, B. (2016). Buenas prácticas en educación Inclusiva. Educatio Siglo XXI, 34 (1), 31-50. http://dx.doi.org/10.6018///25252.

Orden ECI/3854/2007, de 27 de diciembre, por la que se establecen los requisitos para la verificación de los títulos universitarios oficiales que habiliten para el ejercicio de la profesión de Maestro en Educación Infantil. BOE núm.312, de 29 diciembre 2007.

Orden ECI/3857/2007, de 27 de diciembre, por la que se establecen los requisitos para la verificación de los títulos universitarios oficiales que habiliten para el ejercicio de la profesión de Maestro en Educación Primaria. BOE núm.312, de 29 diciembre 2007.

Real Decreto 1630/2006, de 29 de diciembre, por el que se establecen las enseñanzas mínimas del segundo ciclo de Educación infantil. BOE núm. 4, de 4 de enero 2007.

Real Decreto 126/2014, de 28 de febrero, por el que se establece el currículo básico de la Educación Primaria. BOE núm. 52, de 1 de marzo 2014. 